\title{
Discovery of new neutron-moderating materials at ISIS Neutron and Muon Source
}

\author{
Goran Škoro ${ }^{1, *}$, Giovanni Romanelli ${ }^{1}$, Svemir Rudić $^{1}$, Steven Lilley ${ }^{1}$, and Felix Fernandez-Alonso ${ }^{1,2}$ \\ ${ }^{1}$ ISIS Neutron and Muon Source, Science and Technology Facilities Council, Rutherford Appleton Laboratory, Didcot OX11 0QX, \\ United Kingdom \\ ${ }^{2}$ Department of Physics \& Astronomy, University College London, London WC1E 6BT, United Kingdom
}

\begin{abstract}
In this paper, the possibility of performing a comprehensive neutronic characterization of new materials for neutron moderation at the ISIS Neutron and Muon Source using two of its instruments, VESUVIO and TOSCA, will be discussed.
\end{abstract}

\section{Introduction}

The moderator is the most important component of a neutron source. Its role is to slow down neutrons leaked from a target (in the case of accelerator based neutron source) or a reactor to very low energies needed for material science studies. Historically speaking, the very first neutron sources specialized for neutron scattering experiments used thermal neutrons. These days there is increasing interest in the production of cold (and ultracold) neutrons at the neutron sources because of their significant advantages for materials research. Liquid/solid deuterium $\left(D_{2}\right)$, liquid hydrogen $\left(\mathrm{H}_{2}\right)$ and hydrocarbons (for example, liquid/solid methane) are the standard choices of moderator materials at the reactors and accelerator based cold neutron sources. All those materials have very good neutron moderation characteristics but serious disadvantages as well: limited neutron energy range (and relatively low proton density) in the case of liquid hydrogen or limited use of hydrocarbon materials at the high power neutron sources because they are prone to serious radiation damage. As a result of this, the active search for a new types of moderator materials, and especially cryogenic moderator materials, is underway around the world. In this paper, ongoing activities at the ISIS Neutron and Muon Source [1] related to the search for the new neutron-moderating materials are presented.

\section{Characterization of new neutron-moderating materials}

The quality of a potential cold moderator material is practically determined by its excitation spectrum because of its dominant contribution to the energy exchange with the neutron at low temperatures. Also, the cross-section of this material must be measured very accurately in corresponding energy and temperature region. So, one could

\footnotetext{
*e-mail: goran.skoro@stfc.ac.uk
}

say that the way to identify and characterize potentially interesting neutron-moderating materials is to measure their basic neutronic properties: total cross-section and vibrational density of states (VDoS) and use the obtained results for the creation of so-called scattering kernels. These scattering kernels linked with contemporary Monte Carlo radiation transport codes are used then to perform "virtual" experiments and test the effectiveness of new materials for neutron moderation. As an illustration of current capabilities at ISIS to significantly improve and accelerate the process of identification and initial characterization of new neutron-moderating materials, the results of recent measurements of neutronic properties of one of the possible moderator materials, triphenylmethane, using VESUVIO [2] and TOSCA [3] instruments, will be discussed.

\subsection{Triphenylmethane}

Triphenylmethane (TPM), hydrocarbon with the formula $\left(\mathrm{C}_{6} \mathrm{H}_{5}\right)_{3} \mathrm{CH}$ has three aromatic phenyl groups surrounding a central carbon atom (see Figure 1). It is a colorless solid (in powder form) at room temperature with a melting point around $92{ }^{\circ} \mathrm{C}$ and a boiling point around 359 ${ }^{\circ} \mathrm{C}$. Triphenylmethane has been proposed as a material of potential interest as a cold neutron moderator [4] because of its relatively high hydrogen content and relatively low neutron absorption cross-section (5.4 barns/molecule for thermal neutrons). In addition to this, the TPM is capable of forming relatively stable radicals ("self-repairing" effect, similar to water) so the effects of radiation-induced polymerization, creating damaging operational problems for solid and liquid methane moderators (see, for example, Reference [5]), are significantly reduced.

\section{Measurement of the triphenylmethane cross-section}

Initial measurements of the TPM cross-section as a function of temperature have been performed in the Manuel 


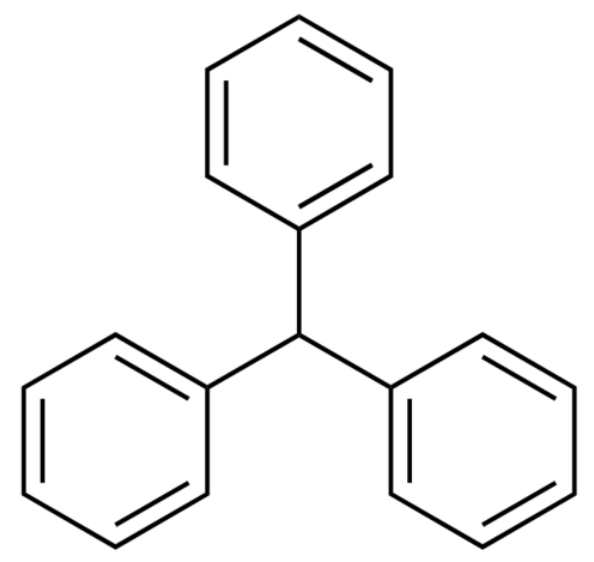

Figure 1. The structure of TPM.

Lujan Jr. Neutron Scattering Center at Los Alamos National Laboratory (LANSCE) [4]. The total cross-section has been measured at room temperature, $100 \mathrm{~K}$ and $10 \mathrm{~K}$. It was found that the TPM cross-section is practically independent of temperature above $30 \mathrm{meV}$. Between 0.3 and $30 \mathrm{meV}$ the cross-section increases with increasing temperature. The most interesting feature, however, of crosssection dependence on energy (for all temperatures) was the presence of a few peaks in the energy range between 7 and $10 \mathrm{meV}$. The authors have explained this unexpected result as a strong indication that the TPM vibrational density of states has very strong modes in this energy range (more details about the TPM vibrational density of states are given below). The total scattering cross-section of triphenylmethane at different temperatures was also measured in a transmission experiment [6] at the Japan Spallation Neutron Source (JSNS) at J-PARC. The shape of the cross-section dependence on neutron energy was similar to the previous result except the fact that measurements at JPARC did not show any peaks in cross-section spectrum. These two conflicting results were additional motivation for preparation of a similar experiment, under the leadership of the team from Centro Atomico Bariloche, CNEA (Argentina), using VESUVIO instrument at ISIS Neutron and Muon Source.

VESUVIO is an indirect-geometry spectrometer (see Figure 2) mainly employed for the determination of nuclear quantum effects in materials using Deep Inelastic Neutron Scattering. However, in recent years, VESUVIO has become an experimental station for epithermal and thermal neutron analysis (VESUVIO+) [8], where, for example, the energy range accessible for neutron transmission experiments (total cross-section determination) spans 8 orders of magnitude, from a fraction of $\mathrm{meV}$ to tens of $\mathrm{keV}$ [9].

Using the protocols described in Reference [10], the total cross-section of triphenylmethane has been measured in a range of temperatures, from room temperature down to $22 \mathrm{~K}$ (see Figure 3 ). The room temperature results were practically identical with the results obtained at J-PARC. The temperature dependence of cross-section showed the

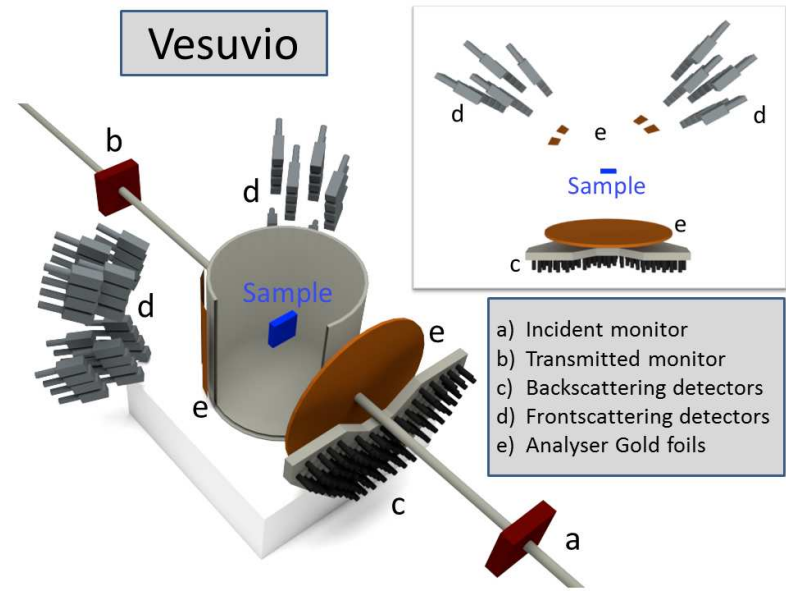

Figure 2. Schematic representation of the VESUVIO spectrometer.

expected trend in the low energy region (as previously observed in the LANSCE experimental data) but ISIS results (similar to the results from J-PARC) did not show any peaks in the cross-section spectra. This is a good example of the need to measure neutronic and/or material properties across facilities. More details about experimental method and cross-section measurements of the TPM at VESUVIO, as well as the description of the procedure for obtaining the corresponding scattering kernel, can be found in Reference [10].

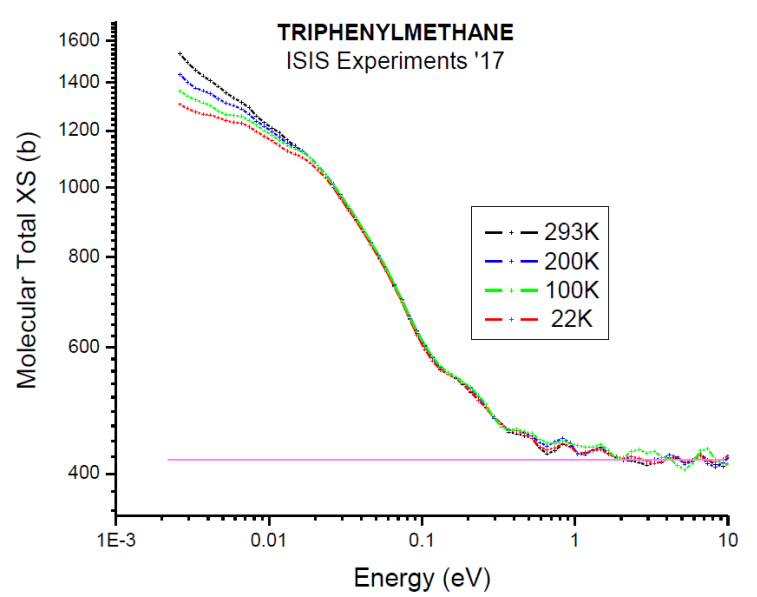

Figure 3. The cross-section of TPM, as measured at VESUVIO instrument, as a function of energy for different temperatures. The solid line represents the asymptotic value of the total cross section based on the free gas values for hydrogen and carbon.

\section{Measurement of the triphenylmethane excitation spectrum}

In order to obtain the VDoS of triphenylmethane, a number of inelastic neutron scattering (INS) experiments were performed using, for example, NERA-PR time-of-flight 


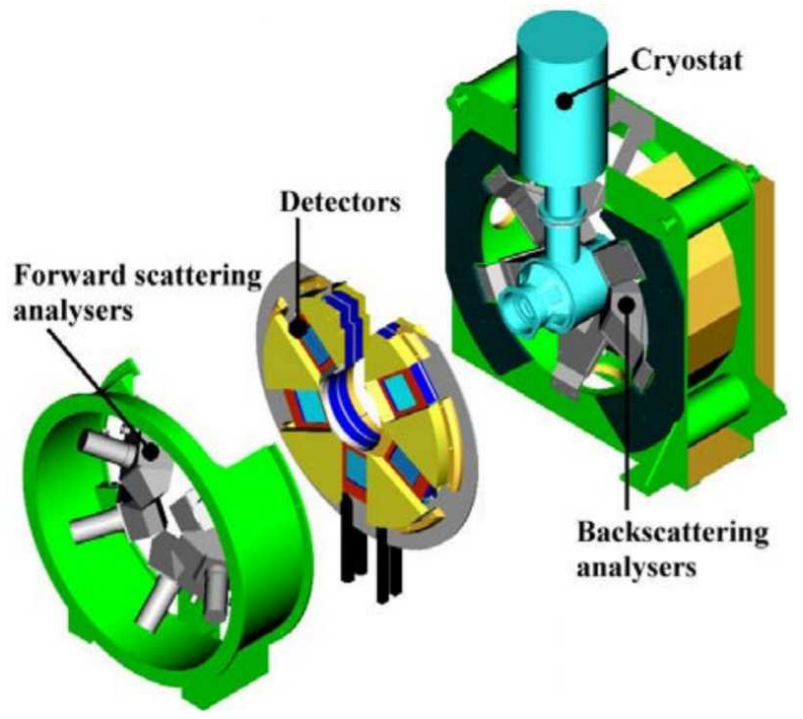

Figure 4. Schematic representation of the TOSCA spectrometer.

spectrometer at pulsed reactor IBR-2 in Dubna [11] and the Filter Difference Spectrometer (FDS) at LANSCE [4].

The same experiment at TOSCA instrument has been proposed with the idea to perform a direct comparison with the results obtained elsewhere to test the instrument capabilities in this field of research. The, recently upgraded [12], TOSCA instrument (see Figure 4) is an indirect-geometry inelastic neutron spectrometer optimized for high resolution vibrational spectroscopy in the energy transfer region between -24 and $4000 \mathrm{~cm}^{-1}$ which makes it, potentially, a very nice tool for the determination of vibrational density of states of moderator material candidates.

The comparison of the TPM inelastic neutron scattering (INS) spectra obtained at three different instruments is presented in Figures 5 - 7. All INS experimental spectra have been measured at cryogenic temperatures (between $10 \mathrm{~K}$ and $22 \mathrm{~K}$ ) and all of them show very high number of excitations. In the low-frequency range, as illustrated in Figure 5, the vibrations of three rings around of central atom appear. Ring deformations dominate in the region between 350 and $1300 \mathrm{~cm}^{-1}$ (see Figures 6 - 7) while stretch vibrations give contribution in the higher frequency region (see Figure 7). The positions of the peaks are, as expected, the same in all the experimental results but it is clearly visible that the TOSCA instrument has superior resolution compared to the NERA-PR and FDS instruments.

It is important to note that such a good resolution is critical for obtaining a high quality characterization of materials, especially those with the very rich excitation spectrum. In the case of TOSCA spectrometer, the resolution $(\Delta E / E)$ is practically constant over full energy transfer range as illustrated in Figure 8.

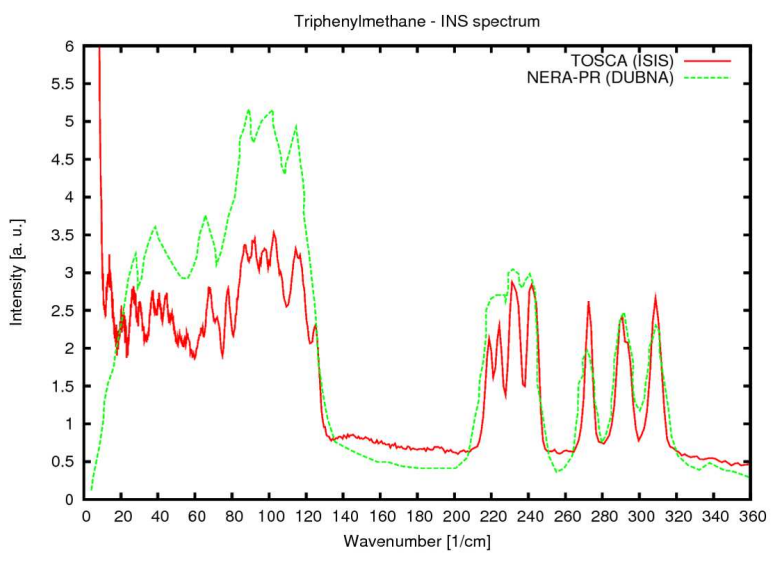

Figure 5. The comparison of the TPM inelastic neutron scattering spectra measured at TOSCA and NERA-PR spectrometer [11] in the energy transfer region between 0 and $350 \mathrm{~cm}^{-1}$.

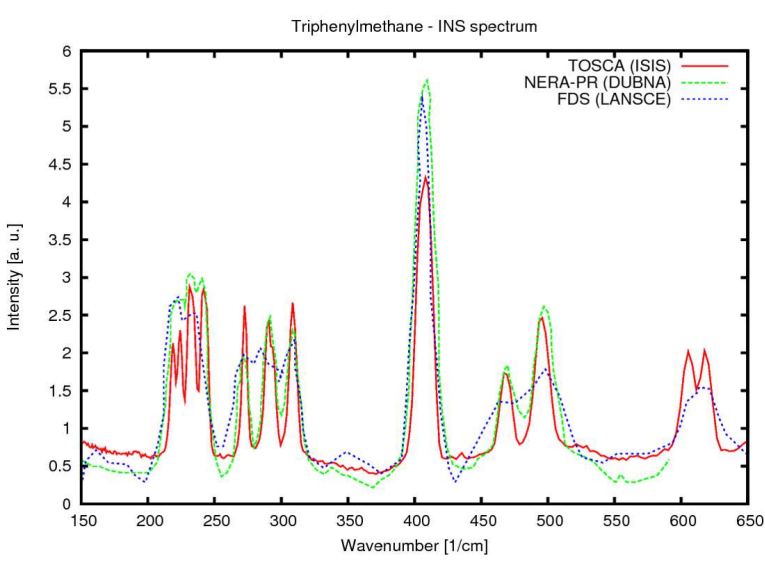

Figure 6. The comparison of the TPM inelastic neutron scattering spectra measured at TOSCA, NERA-PR spectrometer [11] and Filter Difference Spectrometer [4] in the energy transfer region between 150 and $650 \mathrm{~cm}^{-1}$.

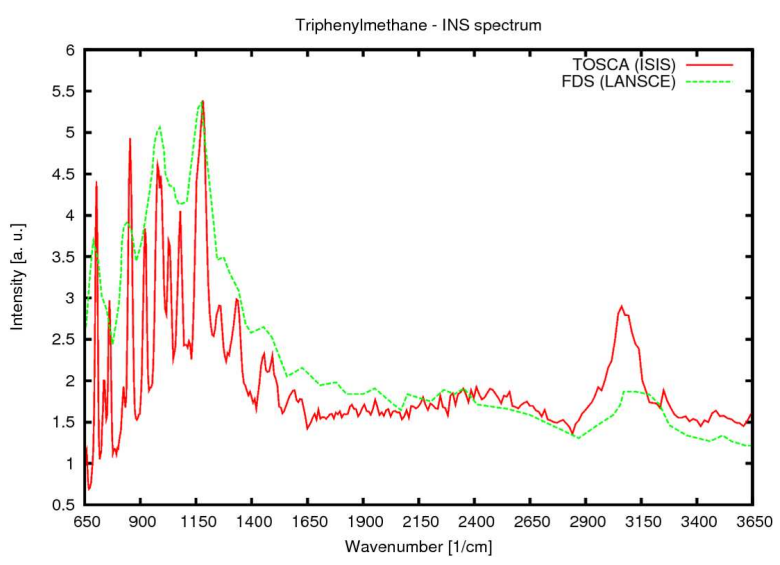

Figure 7. The comparison of the TPM inelastic neutron scattering spectra measured at TOSCA and Filter Difference Spectrometer [4] in the energy transfer region between 650 and 3650 $\mathrm{cm}^{-1}$. 


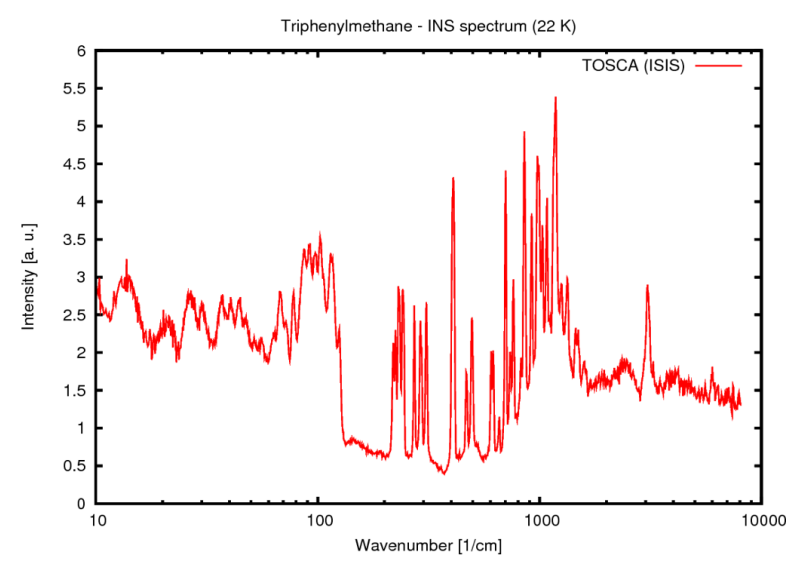

Figure 8. The TPM inelastic neutron scattering spectrum measured at TOSCA. Note that the instrument resolution $(\Delta E / E)$ is practically constant over the full energy transfer range.

\section{Conclusions}

A number of experiments have been performed around the world to measure triphenylmethane cross-sections and vibrational density of states in order to address its use as an efficient moderator material. The high priority of triphenylmethane as a candidate for a new cold moderator material can be illustrated by the fact that its INS spectrum has been very recently measured also at the VISION instrument at Spallation Neutron Source (SNS) [13]. All these measurements across facilities contributed to significant progress in understanding the TPM properties. The obtained results have been used for production of the TPM scattering kernels at cryogenic temperatures [10], in the region where potential advantages of triphenylmethane as a new moderator materials continue to look promising.

Two instruments at ISIS Neutron and Muon Source, VESUVIO and TOSCA, have been also involved in measuring these basic neutronic properties of the TPM. Both of them have produced robust experimental results and showed that the ISIS Neutron and Muon Source has the capability to significantly improve and accelerate the process of identification and initial characterization of new neutron-moderating materials, especially those relevant for efficient production of cold neutrons.

\section{References}

[1] https://www.isis.stfc.ac.uk/Pages/home.aspx.

[2] https://www.isis.stfc.ac.uk/Pages/vesuvio.aspx.

[3] https://www.isis.stfc.ac.uk/Pages/tosca.aspx.

[4] T. Hugle et al., Nuclear Instruments and Methods in Physics Research A 738 1-5 (2014). http://dx.doi.org/10.1016/j.nima.2013.11.063.

[5] D. Evans, Cryogenics 35 163-166 (1995).

[6] M. Teshigawara et al., Talk at Third Research Coordination Meeting (F12026-CR-3) on Advanced Moderators for Intense

[9] Cold Neutron Beams in Materials Research, IAEA, Vienna, 12 - 15 November 2018.

[8] C. Andreani, M. Krzystyniak, G. Romanelli, R. Senesi and F. Fernandez-Alonso, Electronvolt neutron spectroscopy: beyond fundamental systems, Advances in Physics, 2017. http://dx.doi.org/10.1080/00018732.2017.1317963.

[9] G. Romanelli et al., Meas. Sci. Technol. 28095501 (2017). https://doi.org/10.1088/1361-6501/aa7c2a.

[10] F. Cantargi et al., Validated scattering kernels for triphenylmethane at cryogenic temperatures, This Proceedings (2019).

[11] I. Majerz and I. Natkaniec, Physica B 350 e439-e442 (2004). https://doi.org/10.1016/j.physb.2004.03.115.

[12] R. Pinna et al., Nuclear Inst. and Methods in Physics Research, A 896 68-74 (2018). https://doi.org/10.1016/j.nima.2018.04.009.

[13] https://neutrons.ornl.gov/vision. 